\title{
Treatment of adolescents with morbid obesity with bariatric procedures and anti-obesity pharmacological agents
}

This article was published in the following Dove Press journal:

Open Access Surgery

8 December 20II

Number of times this article has been viewed

\author{
Scott S Um' \\ Wendelin Slusser ${ }^{2}$ \\ Daniel A DeUgarte' \\ 'Department of Surgery, David \\ Geffen School of Medicine at UCLA, \\ Los Angeles, CA, USA; '2Department \\ of Pediatrics, David Geffen School of \\ Medicine at UCLA, Los Angeles, \\ CA, USA
}

\begin{abstract}
Adolescent obesity is a growing health concern that can have immense physical and psychological impact. Treatment of morbidly obese adolescents should include a multidisciplinary team to address medical comorbidities, diet, physical activity, mental health, and behavior modification. Anti-obesity pharmacologic agents have a limited role in the treatment of adolescents because of concerns with side effects, safety, and efficacy. Orlistat (GlaxoSmithKline, Moon Township, PA) is the only approved medication for weight-loss in adolescents. However, it is associated with gastrointestinal side effects and its long-term efficacy is unknown. Bariatric surgery is the most effective therapy to treat morbid obesity. However, adolescents must meet rigorous criteria and have appropriate cognitive, psychological, and social clearance before being considered for surgical intervention. Gastric bypass remains the gold standard bariatric operation. The adjustable gastric band is not FDA-approved for use in patients under 18 years of age. Sleeve gastrectomy is a promising procedure for adolescents because it avoids an intestinal bypass and the implantation of a foreign body. Prospective longitudinal assessment of bariatric surgery procedures is required to determine long-term outcomes. In this manuscript, we review the treatment options, efficacy, and impact on quality of life for morbidly obese adolescents.
\end{abstract}

Keywords: bariatric surgery, morbid obesity, weight loss, adolescent

\section{Introduction}

Adolescent obesity is a major public health problem, and it can be associated with multiple comorbidities throughout life. During childhood, obesity is defined as body mass index (BMI) greater than the 95th percentile for age and gender, and it affects $17 \%$ of the US population under 19 years of age. ${ }^{1}$ The prevalence of obesity and obesity-related disease among adolescents in the US has risen dramatically over the past few decades (Figure 1).

Childhood obesity is associated with substantial medical and psychosocial morbidity. ${ }^{2}$ Metabolic syndrome is also increasingly prevalent in adolescents, in whom it is defined by the presence of three of the following: central obesity, glucose intolerance, hypertension, and dyslipidemia. ${ }^{3}$ The health consequences of this epidemic are enormous and the burdens on our health care system are rapidly increasing. ${ }^{4,5}$ It is critical that health professionals appropriately identify, diagnose, and treat adolescent obesity and its associated comorbidities.

Early intervention during adolescence is essential in halting and reversing the serious long-term health consequences of morbid obesity. Diet, exercise, and behavioral therapy are essential for the treatment of the obese adolescent. ${ }^{5,6}$ However, these
Correspondence: Daniel DeUgarte Division of Pediatric Surgery, Box 709818. UCLA Center for Health Sciences, 10833 Le Conte Avenue, Los Angeles, CA, 90095-7098, USA Tel +310 2062429

Fax +310206 II 20

Email ddeugarte@mednet.ucla.edu 


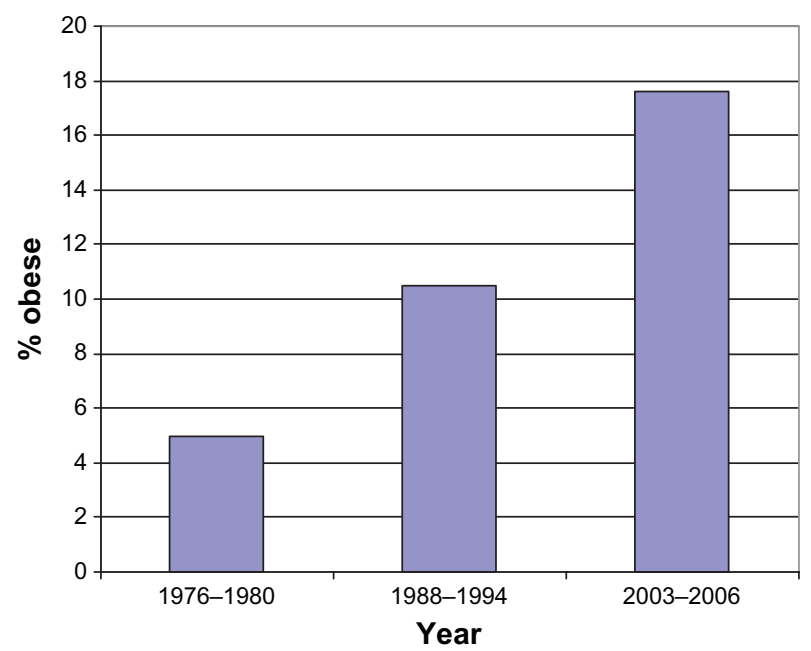

Figure I Prevalence of obesity (sex- and age-specific body mass index $\geq 95$ th percentile) among adolescents (age 12-19 years) in the United States (based on CDC growth charts and data from the National Health and Nutrition Examination Surveys [http://www.education.com/reference/article/obesity-prevalence/?page=3]).'

interventions alone do not often lead to significant weight reduction in the morbidly obese adolescent. Little is known about the long-term benefits and risks of medications used to treat obesity in adolescents, although they may be beneficial in the short term. ${ }^{6}$ Surgical intervention, however, has been shown to lead to the resolution of comorbid conditions such as type 2 diabetes, hypertension, dyslipidemia, obstructive sleep apnea, and liver disease. ${ }^{7,8}$ Comorbidities like nonalcoholic fatty liver disease and diabetes appear to respond better when in the early stages of the disease. ${ }^{9,10}$ For example, islet cell "burn out" can occur in diabetic patients with long-standing disease. ${ }^{10}$ Similarly, advanced nonalcoholic fatty liver disease leading to cirrhosis may no longer be reversible. ${ }^{9}$ Intervention in patients with a lower BMI is likely more effective in reversing comorbidities since they are less advanced. Furthermore, a low BMI can be achieved when intervention is performed at lower preoperative BMIs. ${ }^{11}$ Delaying surgery may result in ongoing weight gain and can impact the "best" weight a patient can achieve. Intervention can also improve life expectancy ${ }^{12,13}$ and quality of life. ${ }^{14,15}$

In the properly selected patient, bariatric surgery is the most effective therapy to treat morbid obesity in adolescents and adults. ${ }^{7,16}$ However, screening and selection for surgical intervention in adolescents can be complicated by factors including patient maturity level, mental health, family dynamics, and social support. In addition, the long-term impact of bariatric surgery on adolescents requires longitudinal assessment.

\section{Review of surgical strategies}

Adolescents with morbid obesity require specialized medical and psychosocial care involving a multidisciplinary team. ${ }^{17}$
The multidisciplinary team consists of a surgeon and/or bariatrician, dietitian, mental health specialist, and bariatric coordinator. While adolescents are commonly treated by pediatricians, they may have chronic diseases more commonly seen in adults. Subspecialists including cardiologists, pulmonologists, gastroenterologists, and endocrinologists should be available for consultation when indicated. Medical work-up should also include an evaluation for depression and other psychiatric disorders. Treatment of depression should be initiated prior to bariatric surgery.

As with adults, adolescents must demonstrate a commitment to lifestyle modifications including adherence to a diet and exercise regimen before being considered for bariatric surgery. Adolescents are required to complete a physiciansupervised behavioral therapy program for a minimum of 6 months. They must be evaluated by a psychologist or psychiatrist with expertise in the psychology of obesity. The comprehensive psychological evaluation involves patient and parent interviews, assessment of the family dynamics, determination of the coping skills of the adolescent, and assessment of the severity of psychosocial comorbidities.

Several laboratory studies are performed to screen for comorbidities as part of the preoperative evaluation. Laboratory tests include fasting glucose and hemoglobin $\mathrm{A}_{1 \mathrm{C}}$, liver function tests, lipid panel, chemistry panel, complete blood count, thyroid function tests, minerals and vitamins, coagulation studies, and a pregnancy test. For patients with obstructive sleep apnea, a sleep study is advisable.

Although bariatric surgery has an extremely low risk of death, ${ }^{18-20}$ both patients and parents must be aware that bariatric surgery is a procedure with potential complications. Postoperatively, strict adherence to dietary regimens and follow-up are paramount. Patients must be highly motivated and capable of understanding the lifestyle changes that are necessary. They must also understand the risks, side effects, and lifelong need for medical surveillance. It is also critical that access to the bariatric team is readily available in order to address the potential short- and long-term consequences of bariatric surgery. For patients under 18 years of age, informed consent is obtained from parents and assent is obtained from the adolescent.

Indications for bariatric surgery in adolescents are similar to adults $^{7}$ (Table 1 ). BMI $>35 \mathrm{~kg} / \mathrm{m}^{2}$ with serious comorbidities or BMI $>40 \mathrm{~kg} / \mathrm{m}^{2}$ are the criteria generally accepted by surgeons and insurance carriers. Serious comorbidities include type 2 diabetes, moderate-to-severe obstructive sleep apnea, severe and/or progressive nonalcoholic steatohepatitis (NASH), and pseudotumor cerebri. Other indications include 
Table I Indications for bariatric surgery in adolescents

BMI $\geq 35$ with serious comorbidities (type 2 diabetes mellitus, moderate or severe obstructive sleep apnea, pseudotumor cerebri, and severe steatohepatitis)

$\mathrm{BMI} \geq 40$ with other comorbidities (hypertension, insulin resistance, glucose intolerance, impaired quality of life or activities of daily living, dyslipidemia, sleep apnea).

General requirements

- Physician-supervised behavioral therapy (>6 months) with demonstrated commitment to follow-up and evidence of diet and physical activity changes.

- Skeletal maturity and Tanner Stage IV/V (if a malabsorptive procedure will be performed).

- Psychiatric/psychological clearance.

- Cognitive maturity (informed assent for patients under 18 years of age).

- Appropriate family/social support.

mild obstructive sleep apnea, mild NASH, hypertension, dyslipidemia, and impaired quality of life. Skeletal maturity (completion of $95 \%$ of adult stature on radiographic study) should be achieved prior to performing malabsorptive procedures, given the potentially higher risks of nutrient and vitamin deficiencies and impact on growth and development. In general, the minimum recommended age for girls is 13 years and for boys is 15 years.

There are three bariatric operations that are routinely performed in the US in adults and adolescents: gastric bypass, gastric banding, and sleeve gastrectomy (Figure 2). All procedures are now performed routinely using a laparoscopic approach. Procedures have been historically divided mechanistically into three types: restrictive, malabsorptive, and combined restrictive/malabsorptive. Restrictive procedures are those which promote early satiety by decreasing the capacity of the stomach or pouch. Malabsorptive procedures involve bypassing a segment of intestine to minimize absorption. Roux-en-Y gastric bypass remains the gold standard for long-term weight loss. ${ }^{7}$ It is both a restrictive and malabsorptive procedure (Figure 2A). The stomach is divided with a stapler to create a small gastric pouch. Intestinal bypass is achieved with a 100-150 cm Roux jejunal limb. Potential complications of this procedure include dumping syndrome, malabsorption, mineral deficiency, marginal ulcer, stomal stenosis, and internal hernia. ${ }^{21}$ Gastric bypass in adolescents appears to be more effective than gastric banding in weight loss and resolution of comorbidities. ${ }^{8}$ The mean excess body weight loss is $>60 \%$. The major disadvantage of gastric bypass is the malabsorption caused by intestinal bypass and the lifelong need for nutritional supplementation. Bypass can also prevent endoscopic surveillance of the bypassed foregut. Potential short-term complications of bypass include anastomotic leak, bleeding, and thromboembolic disease. Potential longterm complications include bowel obstruction, symptomatic cholelithiasis, and micronutrient deficiencies (eg, iron, calcium, and vitamin B12).

Adjustable gastric banding is a restrictive procedure that is less invasive, potentially reversible, and requires a shorter hospitalization (Figure 2B). ${ }^{22-25}$ It involves placing an adjustable band around the stomach that restricts the passage of food. The band tightness is adjusted by needle injection of fluid into a port that is located in the subcutaneous tissue. Frequent adjustments are often required to optimize weight loss without causing vomiting. Utilization of gastric banding in adolescents has been increasing despite the lack of FDA (Food and Drug Administration) approval for patients under 18 years of age. ${ }^{19,26}$ Complications of the procedure include gastric erosion, band slippage or displacement, port malfunction or infection, and gastric pouch or esophageal dilation. ${ }^{21}$ These complications often require operative revision or removal. Results of gastric banding are generally inferior to gastric bypass. ${ }^{7,8}$ The durability of gastric banding in younger patients has also been questioned because it involves insertion of a foreign body, which is likely to malfunction and ultimately require replacement or removal. ${ }^{23,24}$

In the US, sleeve gastrectomy has become increasingly popular in the last few years. This promising procedure is a restrictive procedure that involves resection of approximately $70 \%$ of the stomach (Figure $2 \mathrm{C}$ ). The procedure avoids intestinal bypass. Its long-term weight loss is comparable to that of gastric bypass based on recent short-term studies. ${ }^{27}$ In addition to producing satiety by reducing the capacity of the stomach, sleeve gastrectomy is associated with decreased levels of ghrelin (an orexigenic hormone that is produced in the fundus) resulting in appetite suppression and early satiety. ${ }^{28}$ Unlike gastric banding, no foreign body is implanted and there is no need for adjustments with sleeve gastrectomy.

Biliopancreatic diversion and duodenal switch is both a restrictive and malabsorptive procedure that is performed in adults but not widely used in the adolescent population. ${ }^{7}$ The high-risk profile and potential for significant malabsorption makes the procedure difficult to justify in adolescents.

\section{Review of pharmacologic strategies}

Anti-obesity pharmacologic agents are widely used in obese adults. However, in obese adolescents, their use is often limited to research protocols. ${ }^{29}$ The safety and efficacy of these agents in obese adolescents is unclear. 
There are various anti-obesity pharmacologic agents on the market. Any anti-obesity drug treatment is only an adjunct to diet and activity therapies. Currently, there are several FDA-approved drugs available for the treatment of obesity in adults. However, only one, Orlistat, is approved for use in children and adolescents. ${ }^{6}$ Sibutramine (Abbott
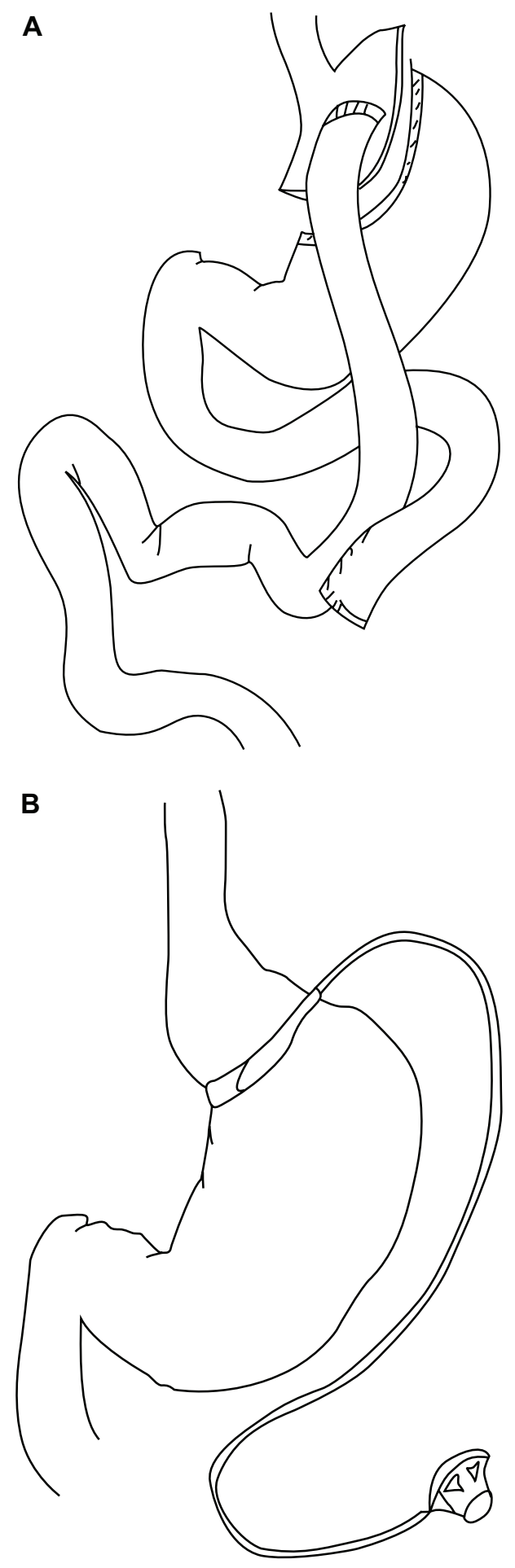

Figure 2 (Continued)

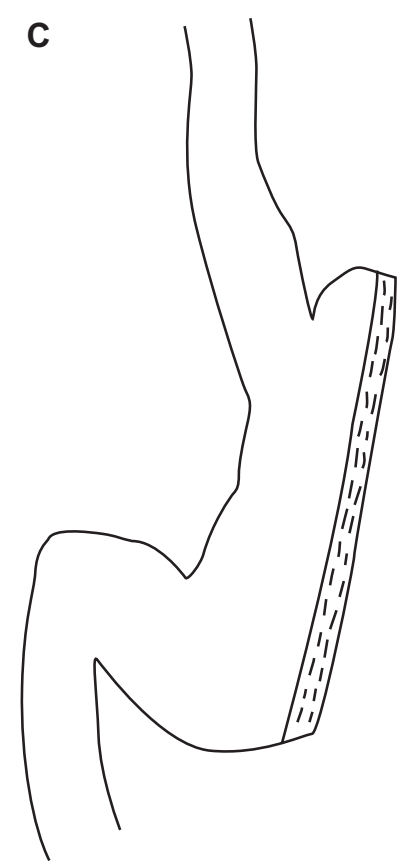

Figure 2 Bariatric surgery options for adolescents. (A) Gastric bypass involves creation of a restrictive gastric pouch and intestinal bypass with a $100-150 \mathrm{~cm}$ Roux jejunal limb. (B) Gastric banding utilizes a device around the proximal stomach to adjustably restrict passage of food. The band tightness is adjusted using a subcutaneous port to infuse or withdraw fluid. Devices are not currently FDA-approved for patients under 18 years of age. (C) Sleeve gastrectomy involves resection of the greater curvature of the stomach to create a tube-shaped stomach.

Laboratories, North Chicago, IL) was withdrawn from the market in October 2010.

Orlistat is a pancreatic lipase inhibitor that reduces fat absorption from the gastrointestinal tract. It has been effective in both adults and adolescent clinical trials in reducing weight. ${ }^{30}$ It was approved by the FDA in 2003 for adolescents 12 years or older. ${ }^{17}$ It can have significant gastrointestinal side effects such as flatulence, steatorrhea, and malabsorption, especially if dietary fat intake is much more than $30 \%$ of total daily caloric intake. A daily multivitamin that contains fat-soluble vitamins is recommended while taking this drug.

Sibutramine is a centrally acting agent that reduces appetite. It inhibits the reuptake of serotonin and norepinephrine and, to some degree, dopamine. The drug was approved by the FDA for patients 16 years or older, but it was withdrawn from the market in October 2010 due to risks of serious cardiovascular events. ${ }^{31}$

No pharmacologic agent has been proven effective in achieving long-term weight loss after medication is stopped. The side effects of these pharmacologic drugs are significant, and long-term trials are required for further evaluation. 


\section{Relative effectiveness}

Behavioral interventions that focus on obese adolescents and families generally have low success in achieving long-term weight loss. ${ }^{32,33}$ Short-term weight loss has been achieved in randomized, controlled trials involving various strategies including diet, control, and exercise. . $2,34,35^{-35}$ These strategies include calorie and fat reduction, low carbohydrate diet, reduction of sedentary behaviors, and participation in structured, vigorous physical activity. In adolescents, lifestyle intervention can result in a decrease in BMI of 3 units at 1-year follow-up. ${ }^{35}$ The results from a meta-analysis reviewing patients ages 4 to 18 years enrolled in comprehensive behavioral interventions (weight loss or healthy diet counseling, physical activity counseling or activity program, and behavioral management techniques) showed that the medium-to-high intensity programs (having 26 hours to over 75 hours of total intervention time) can be effective over the short term (up to 12 months after intervention). ${ }^{6}$

Bariatric surgery has proven to be safe and effective in the treatment of morbid obesity as it leads to significant long-term weight loss and the rapid resolution of comorbidities. ${ }^{7,16,36}$ It is also associated with an increased life expectancy. ${ }^{12,13}$ It has been proposed that procedures that involve duodenal bypass provide better rates of diabetes remission. After gastric bypass, the majority of patients with type 2 diabetes are off medications with dramatic improvements in vital signs, hemoglobin $\mathrm{A}_{1 \mathrm{C}}$, and lipid profiles. ${ }^{16,37}$ In adolescents, a meta-analysis of six studies on gastric bypass showed nearly complete resolution of hypertension and sleep apnea along with $>35 \%$ reduction of BMI. ${ }^{8}$ In one prospective multicenter study, dramatic improvement in lipid profile and reduction of BMI was seen when compared to a nonsurgical cohort (21 units vs 1.2 units)..$^{38}$

A recent Australian randomized controlled trial comparing adjustable gastric banding and a control group (undergoing a program of lifestyle modification) in morbidly obese adolescents demonstrated a 2-year excess body weight loss of $79 \%$ (compared with $13 \%$ in the control group). ${ }^{22}$ However, the operative patients required frequent follow-up for band adjustments, and there was a 33\% reoperation rate which included revisions for band migration, proximal pouch dilation, port-related problems, and infection. In European studies with long-term follow-up, gastric banding results have been worse in younger patients. ${ }^{23,24}$ Compared to gastric bypass, gastric banding is inferior in terms of weight loss. ${ }^{7,8}$ Gastric banding is also thought to have fewer early complications, but a higher rate of late complications. ${ }^{39}$
Complications include gastric erosion, band slippage, and pouch dilation.

Sleeve gastrectomy has gained interest recently due to the fact that it is relatively simple to perform, and good mid-term results have been reported in adults. Recent reviews report $33 \%-85 \%$ excess weight loss, an average complication rate of $6.2 \%$, and an overall mortality of $0.17 \%$ in adult patients. ${ }^{40}$ However, long-term data are limited in both adults and adolescents. ${ }^{41}$ Most mid-term results in adults demonstrate results that are nearly equivalent to gastric bypass and superior to adjustable gastric banding in terms of weight loss and resolution of comorbidities..$^{27,42}$ The main concern is its irreversibility and the potential development of early complications including staple-line bleed and leak.

\section{Patient-focused perspectives (quality of life and long-term nutrition)}

Obesity can significantly impair psychosocial well-being. In one study, obese children were shown to have a quality of life comparable to children with cancer. ${ }^{43}$ The degree of obesity is directly related to impairments in emotional, social, and physical functions. Morbidly obese adolescents experience many problems at school including the social stigma of their weight and physical limitations in various activities. Many are home-schooled because they do not integrate well into the high school environment. Binge eating and self-induced purging also occur in 5\%-30\% of obese adolescents seeking bariatric surgery. ${ }^{7}$ Adolescents who present for bariatric surgery often suffer from depression. ${ }^{15}$ For example, $53 \%$ of adolescents were mildly depressed, $30 \%$ self-reported clinically significant depressive symptoms, and $45 \%$ were clinically depressed based on their mothers' reports. ${ }^{15}$

Bariatric surgery has been demonstrated to significantly improve quality of life in morbidly obese adolescents. ${ }^{14,15}$ Quality of life scores of patients after gastric bypass were significantly better than adolescents with behavioral therapy for obesity. ${ }^{14}$ This was based on the questionnaire addressing physical function, self-esteem, sexual life, public distress, and work. Also patients that had the worst quality of life preoperatively had the most improvement postoperatively. Similarly, significant improvement in overall and weightrelated quality of life was observed after gastric bypass at the 1-year follow-up. ${ }^{15}$ These patients showed improvement in physical comfort, physical capabilities, and body esteem, as well as overall psychosocial health.

Nutritional deficiencies are a potential concern following bariatric surgery, and patients need at minimum yearly 
laboratory tests and close follow-up. Strict adherence to multivitamin and mineral supplementation is critical in preventing nutritional deficiencies. Patients often have low levels of iron, vitamin B12, vitamin D, and calcium after gastric bypass. ${ }^{44,45}$ Adolescents may also be at risk for thiamine deficiency resulting in beriberi. ${ }^{46}$ Noncompliance with medical regimens is a major concern, particularly in adolescents. Therefore, adolescents undergoing bariatric surgery must be carefully assessed for ability to comply with medical regimens and follow-up care.

\section{Conclusion}

Adolescent obesity remains a major public health problem that can lead to major health risks throughout life. Timely intervention can halt the progression of, or reverse, comorbid conditions, significantly improve quality of life, and potentially increase life expectancy. Anti-obesity pharmacological agents have a limited role in the treatment of adolescents due to concerns regarding side effects, safety, and efficacy. In the properly selected patient, bariatric surgery is superior to behavioral therapy alone in the treatment of morbidly obese adolescents. A multidisciplinary team is required to provide behavioral therapy, assess patient motivation and compliance, and provide ongoing support. The gold standard procedure is laparoscopic gastric bypass. The laparoscopic adjustable gastric banding device is not yet approved by the FDA for adolescents $<18$ years of age. Sleeve gastrectomy is a promising procedure for adolescents because it avoids intestinal bypass and implantation of a foreign body. Prospective longitudinal assessment of pharmacologic agents and bariatric surgery procedures is required to determine long-term outcomes.

\section{Disclosure}

The authors report no conflicts of interest in this work.

\section{References}

1. Ogden CL, Carroll MD, Curtin LR, Lamb MM, Flegal KM. Prevalence of high body mass index in US children and adolescents, 2007-2008. JAMA. 2010;303(3):242-249.

2. Franks PW, Hanson RL, Knowler WC, Sievers ML, Bennett PH, Looker HC. Childhood obesity, other cardiovascular risk factors, and premature death. $N$ Engl J Med. 2010;362(6):485-493.

3. Zimmet P, Alberti G, Kaufman F, et al. The metabolic syndrome in children and adolescents. Lancet. 2007;369(9579):2059-2061.

4. Trasande L, Liu Y, Fryer G, Weitzman M. Effects of childhood obesity on hospital care and costs, 1999-2005. Health Aff (Millwood). 2009; 28(4):w751-w760.

5. Slusser W, Staten K, Stephens K, et al. Payment for obesity services: examples and recommendations for stage 3 comprehensive multidisciplinary intervention programs for children and adolescents. Pediatrics. 2011;128 Suppl 2:S78-S85.
6. Whitlock EP, O'Connor EA, Williams SB, Beil TL, Lutz KW. Effectiveness of weight management interventions in children: a targeted systematic review for the USPSTF. Pediatrics. 2010;125(2): e396-e418.

7. Pratt JS, Lenders CM, Dionne EA, et al. Best practice updates for pediatric/adolescent weight loss surgery. Obesity (Silver Spring). 2009; 17(5):901-910

8. Treadwell JR, Sun F, Schoelles K. Systematic review and meta-analysis of bariatric surgery for pediatric obesity. Ann Surg. 2008;248(5): 763-776.

9. Mummadi RR, Kasturi KS, Chennareddygari S, Sood GK. Effect of bariatric surgery on nonalcoholic fatty liver disease: systematic review and meta-analysis. Clin Gastroenterol Hepatol. 2008;6(12): 1396-1402.

10. Schauer PR, Burguera B, Ikramuddin S, et al. Effect of laparoscopic Roux-en Y gastric bypass on type 2 diabetes mellitus. Ann Surg. 2003; 238(4):467-484, discussion 484-465.

11. Inge $\mathrm{TH}$, Jenkins $\mathrm{TM}$, Zeller $\mathrm{M}$, et al. Baseline $\mathrm{BMI}$ is a strong predictor of nadir BMI after adolescent gastric bypass. J Pediatr. 2010;156(1): 103-108, e101.

12. Adams TD, Gress RE, Smith SC, et al. Long-term mortality after gastric bypass surgery. $N$ Engl J Med. 2007;357(8):753-761.

13. Sjostrom L, Narbro K, Sjostrom CD, et al. Effects of bariatric surgery on mortality in Swedish obese subjects. N Engl J Med. 2007;357(8): 741-752.

14. Loux TJ, Haricharan RN, Clements RH, et al. Health-related quality of life before and after bariatric surgery in adolescents. J Pediatr Surg. 2008;43(7):1275-1279.

15. Zeller MH, Modi AC, Noll JG, Long JD, Inge TH. Psychosocial functioning improves following adolescent bariatric surgery. Obesity (Silver Spring). 2009;17(5):985-990.

16. Maggard MA, Shugarman LR, Suttorp M, et al. Meta-analysis: surgical treatment of obesity. Ann Intern Med. 2005;142(7):547-559.

17. Barlow SE. Expert committee recommendations regarding the prevention, assessment, and treatment of child and adolescent overweight and obesity: summary report. Pediatrics. 2007;120 Suppl 4: S164-S192.

18. Schilling PL, Davis MM, Albanese CT, Dutta S, Morton J. National trends in adolescent bariatric surgical procedures and implications for surgical centers of excellence. J Am Coll Surg. 2008;206(1):1-12.

19. Jen HC, Rickard DG, Shew SB, et al. Trends and outcomes of adolescent bariatric surgery in California, 2005-2007. Pediatrics. 2010;126(4):e746-e753.

20. Flum DR, Belle SH, King WC, et al. Perioperative safety in the longitudinal assessment of bariatric surgery. NEngl J Med. 2009;361(5): 445-454.

21. Colquitt JL, Picot J, Loveman E, Clegg AJ. Surgery for obesity. Cochrane Database Syst Rev. 2009;2:CD003641.

22. O'Brien PE, Sawyer SM, Laurie C, et al. Laparoscopic adjustable gastric banding in severely obese adolescents: a randomized trial. JAMA. 2010;303(6):519-526.

23. Lanthaler M, Sieb M, Strasser S, Weiss H, Aigner F, Nehoda H. Disappointing mid-term results after laparoscopic gastric banding in young patients. Surg Obes Relat Dis. 2009;5(2):218-223.

24. Mittermair R, Aigner F, Obermuller S. High complication rate after Swedish adjustable gastric banding in younger patients $<$ or $=25$ years. Obes Surg. 2009;19(4):446-450.

25. Nadler EP, Youn HA, Ren CJ, Fielding GA. An update on 73 US obese pediatric patients treated with laparoscopic adjustable gastric banding: comorbidity resolution and compliance data. J Pediatr Surg. 2008; 43(1):141-146

26. Hinojosa MW, Varela JE, Parikh D, Smith BR, Nguyen XM, Nguyen NT. National trends in use and outcome of laparoscopic adjustable gastric banding. Surg Obes Relat Dis. 2009;5(2):150-155.

27. Brethauer SA, Hammel JP, Schauer PR. Systematic review of sleeve gastrectomy as staging and primary bariatric procedure. Surg Obes Relat Dis. 2009;5(4):469-475. 
28. Langer FB, Reza Hoda MA, Bohdjalian A, et al. Sleeve gastrectomy and gastric banding: effects on plasma ghrelin levels. Obes Surg. 2005; 15(7):1024-1029.

29. Greydanus DE, Bricker LA, Feucht C. Pharmacotherapy for obese adolescents. Pediatr Clin North Am. 2011;58(1):139-153, xi.

30. Freemark M, Kiess W. Antiobesity medication use in adolescents: risks and benefits. Pediatr Endocrinol Rev. Nov 2004;2 Suppl 1: 168-170.

31. Powell AG, Apovian CM, Aronne LJ. New drug targets for the treatment of obesity. Clin Pharmacol Ther. 2011;90(1):40-51.

32. Epstein LH, Valoski A, Wing RR, McCurley J. Ten-year outcomes of behavioral family-based treatment for childhood obesity. Health Psychol. 1994;13(5):373-383.

33. Levine MD, Ringham RM, Kalarchian MA, Wisniewski L, Marcus MD. Is family-based behavioral weight control appropriate for severe pediatric obesity? Int J Eat Disord. 2001;30(3):318-328.

34. Epstein LH, Myers MD, Raynor HA, Saelens BE. Treatment of pediatric obesity. Pediatrics. 1998;101(3 Pt 2):554-570.

35. Oude Luttikhuis H, Baur L, Jansen H, et al. Interventions for treating obesity in children. Cochrane Database Syst Rev. 2009;1: CD001872.

36. Tsai WS, Inge TH, Burd RS. Bariatric surgery in adolescents: recent national trends in use and in-hospital outcome. Arch Pediatr Adolesc Med. 2007;161(3):217-221.

37. Inge TH, Miyano G, Bean J, et al. Reversal of type 2 diabetes mellitus and improvements in cardiovascular risk factors after surgical weight loss in adolescents. Pediatrics. 2009;123(1):214-222.
38. Lawson ML, Kirk S, Mitchell T, et al. One-year outcomes of Roux-en-Y gastric bypass for morbidly obese adolescents: a multicenter study from the Pediatric Bariatric Study Group. J Pediatr Surg. 2006;41(1):137-143.

39. Franco JV, Ruiz PA, Palermo M, Gagner M. A review of studies comparing three laparoscopic procedures in bariatric surgery: sleeve gastrectomy, Roux-en-Y gastric bypass and adjustable gastric banding. Obes Surg. 2011;21(9):1458-1468.

40. Aggarwal S, Kini SU, Herron DM. Laparoscopic sleeve gastrectomy for morbid obesity: a review. Surg Obes Relat Dis. 2007;3(2):189-194.

41. Till HK, Muensterer O, Keller A, et al. Laparoscopic sleeve gastrectomy achieves substantial weight loss in an adolescent girl with morbid obesity. Eur J Pediatr Surg. 2008;18(1):47-49.

42. Himpens J, Dapri G, Cadiere GB. A prospective randomized study between laparoscopic gastric banding and laparoscopic isolated sleeve gastrectomy: results after 1 and 3 years. Obes Surg. 2006;16(11): 1450-1456.

43. Schwimmer JB, Burwinkle TM, Varni JW. Health-related quality of life of severely obese children and adolescents. JAMA. 2003;289(14): 1813-1819.

44. Alvarez-Leite JI. Nutrient deficiencies secondary to bariatric surgery Curr Opin Clin Nutr Metab Care. 2004;7(5):569-575.

45. Halverson JD. Vitamin and mineral deficiencies following obesity surgery. Gastroenterol Clin North Am. 1987;16(2):307-315.

46. Towbin A, Inge TH, Garcia VF, et al. Beriberi after gastric bypass surgery in adolescence. J Pediatr. 2004;145(2):263-267.
Open Access Surgery

\section{Publish your work in this journal}

Open Access Surgery is an international, peer-reviewed, open access journal that focuses on all aspects of surgical procedures and interventions. Patient care around the peri-operative period and patient outcomes post surgery are key topics. All grades of surgery from minor cosmetic interventions to major surgical procedures are covered. Novel techniques

\section{Dovepress}

and the utilization of new instruments and materials, including implants and prostheses that optimize outcomes constitute major areas of interest. The manuscript management system is completely online and includes a very quick and fair peer-review system. Visit http://www.dovepress.com/ testimonials.php to read real quotes from published authors. 